\title{
¿Predicen los factores de depresión, ansiedad y estrés la dimensión de la despersonalización y la baja realización personal en el profesorado?
}

\author{
Lucía Granados ${ }^{2}$, David Aparisi ${ }^{1}$, Cándido J. Inglés ${ }^{3}$, \\ María del Pilar Aparicio-Flores ${ }^{1}$, Aitana Fernández-Sogorb ${ }^{1}$ y \\ José M. García-Fernández ${ }^{1}$ \\ ${ }^{1}$ Universidad de Alicante (España); ${ }^{2}$ Universidad Internacional de Valencia (España); \\ ${ }^{3}$ Universidad Miguel Hernández, Elche (España)
}

\begin{abstract}
En los últimos años, la investigación en burnout está en aumento debido a la relación con problemas físicos y mentales. Especialmente, la enseñanza ha sido una de las profesiones más expuesta al estudio de las condiciones laborales y los efectos que conlleva. El objetivo de este trabajo fue analizar, mediante análisis de regresión logística, si la Depresión, la Ansiedad y el Estrés son predictores de altos niveles de Despersonalización y baja Realización Personal en docentes no-universitarios. La muestra final se compuso de 634 profesores $(\mathrm{N}=376$ hombres y 258 mujeres). El rango de edad para la muestra total fue de 29 a 65 años ( $M$ edad=45.81; $D T=13.35)$. Los instrumentos utilizados fueron, Escalas de Depresión, Ansiedad y Estrés -21 (DASS-21) y el Maslach Burnout Inventory (MBI). Los resultados indicaron que la probabilidad de presentar alta Despersonalización y baja Realización Personal es mayor por cada punto de aumento en las variables Depresión, Ansiedad y Estrés. En conclusión, los hallazgos de este estudio revelan un avance en el estudio de la Despersonalización y la baja Realización Personal en profesionales de la enseñanza.
\end{abstract}

Palabras clave: Depresión, ansiedad, estrés, despersonalización, baja realización personal.

Do the depression, anxiety and stress factors predict the dimension of depersonalization and low personal accomplishment in professorate? In the last years, the research in burnout is increasing due to its physical and mental problems relationship. Concretely, teaching has been one of the most exposed professions to the study of the working conditions and the effects it carries on. The aim of this work was to analyze, by means of logistic regression analysis, if Depression, Anxiety and Stress are predictors of high levels of Depersonalization and low Personal Accomplishment in teaching staff out of the university field. The final sample consisted of 634 teachers $(\mathrm{N}=376$ men and 258 women). The age range for the total sample was from 29 to 65 years ( $M$ age $=45.81$, $S D=13.35$ ). The instruments used were Depression, Anxiety and Stress Scales-21 (DASS-21) and the Maslach Burnout Inventory (MBI). Results indicated that the probability of presenting high Depersonalization and low Personal Accomplishment are greater for each increasing point in the Depression, Anxiety and Stress variables. In conclusion, the findings of this study reveal an advance in the study of Depersonalization and low Personal Accomplishment in education professionals.

Keywords: Depression, anxiety, stress, depersonalization, low personal accomplishment.

Correspondencia: María del Pilar Aparicio Flores. Departamento de Psicología y Didáctica. Facultad de Educación. Universidad de Alicante. Apdo. Correos, 99. C.P.: 03080. Alicante (España). E-mail:pilar.aparicio@ua.es 
La enseñanza ha sido la profesión más expuesta al estudio de las condiciones laborales (Llorca-Rubio y Gil-Monte, 2014). Muchos han sido los estudios que han investigado las condiciones laborales de la profesión docente y sus efectos físicos y mentales (Cladellas y Castelló, 2011).

Pese a la profusión de investigaciones sobre la salud y el estrés laboral docente, los resultados siguen siendo bastante complejos de integrar, debido a que muchos de los estudios se han centrado en conceptos como el desgaste profesional o burnout (Vera, Salanova, y Martín, 2010), síndrome que parece surgir como respuesta a un continuado estrés laboral (Figueiredo-Ferraz, Gil-Monte, Grau-Alberola, Llorca-Pellicer, y García-Juesas, 2012) y que puede afectar a la salud física y mental y a las relaciones sociales de los trabajadores que lo padecen (Grau, Suñer, y García, 2005).

Diversas investigaciones muestran la importancia del estudio del estrés y la evaluación de autocontrol ante las demandas sociales, por su perjudicial gran poder sobre la salud física y el bienestar emocional. Ejemplos de ello son el riesgo hacia enfermedades cardiovasculares, hipertensión, asma, jaquecas, dolores musculares, y depresión, entre otros problemas de salud (Cockerham, 2001; Mechanic, 1976; Siegrist, Siegrist, y Weber, 1986; Turner, Wheaton, y Lloyd, 1995).

Por su parte, Salanova, Llorens, y García-Renedo (2003), encontraron que los estresores que consideran más importantes los docentes son, en orden de mayor a menor importancia, el exceso de trabajo, nivel emocional (por la exigencia de implicarse a nivel personal con los alumnos, padres, y sociedad en situaciones conflictivas), ambigüedad y conflicto de rol, falta de apoyo social por parte de compañeros y/o del centro, falta de coordinación para realizar el trabajo en equipo, desmotivación, desgana, e indisciplina del alumnado. Estos hallazgos coinciden con un estudio evaluado con docentes venezolanos ( $\mathrm{N}=885$ maestros), realizado por Oramas-Viera, Almirall-Hernández, y Fernández (2007), cuyos participantes añadieron además el salario inadecuado y la carencia de recursos personales y materiales.

Teniendo en cuenta la literatura previa, existe una gran evidencia que respalda la relación entre condiciones de trabajo y salud laboral, productividad y permanencia en el empleo por parte de trabajadores (Cornejo-Chávez y Quiñónez, 2007).

Sin embargo, existe una carencia de trabajos que analicen la relación entre los niveles de Depresión, Ansiedad y Estrés, y las diferentes dimensiones del burnout, en concreto, la Despersonalización y la baja Realización Personal, entendidas estas como una actitud distante, de insensibilidad emocional y de carencia empática ante la sociedad, por lo que respecta a la Despersonalización, y el sentimiento de reducción de capacidad personal y, consecuentemente, insatisfacción por el auto-rendimiento profesional, por lo que respecta a la baja Realización Personal (Díaz-Bambula, López-Sánchez, y VarelaArévalo, 2012). En este sentido es de relevante importancia su estudio, teniendo en cuenta investigaciones previas que predicen el Estrés entre docentes como un posible agotamiento 
emocional (Skaalvik y Skaalvic, 2011; 2016), la Ansiedad como una amenaza en el bienestar personal del profesorado (Frenzel et al., 2016) y una asociación positiva y significativa entre Estrés, Ansiedad y Depresión evaluada con docentes (Husain, Gulzar, Aqueel, y Rana, 2016).

\section{Objetivo}

El presente estudio tuvo como objetivo analizar, mediante análisis de regresión logística, si la Depresión, la Ansiedad y el Estrés son predictores de altos niveles de Despersonalización y baja Realización Personal en personal docente español no universitario.

\section{MÉTODO}

\section{Participantes}

La muestra estuvo compuesta por 634 profesores de Educación Infantil (EI), Educación Primaria (EP) y Educación Secundaria Obligatoria (ESO) (N=376 hombres y 258 mujeres), pertenecientes a colegios concertados de las provincias de Alicante, Murcia y Valencia. El rango de edad para la muestra total fue de 20 a 65 años $(M$ edad=45.81; $D T=13.35$ ).

Tabla 1. Número y porcentaje de sujetos, subtotales y totales en términos de sexo y edad

\begin{tabular}{lccccccc}
\hline & \multicolumn{3}{c}{ Masculino } & & \multicolumn{2}{c}{ Femenino } & \multicolumn{3}{c}{ Total } \\
\hline Variables & $n$ & $\%$ & $n$ & $\%$ & & $\%$ \\
\hline$N$ Total 634 & 376 & 51.1 & 258 & 42.9 & 634 & 100 \\
\hline $20-29$ & 42 & 11.1 & 34 & 13.1 & 76 & 11.9 \\
\hline & & \multicolumn{2}{c}{ Edad } & & & \\
\hline $30-39$ & 85 & 22.6 & 69 & 26.7 & 154 & 24.2 \\
\hline $40-49$ & 141 & 37.5 & 86 & 33.3 & 227 & 35.8 \\
\hline$\geq 50$ & 108 & 28.7 & 69 & 26.7 & 177 & 279 \\
\hline
\end{tabular}

La tabla 1 recoge el análisis descriptivo referente a la edad de los participantes, en la que se destaca una proporción equivalente, en ambos sexos, respecto a profesionales con edades comprendidas entre 40 y 49 años, y más de 50 años. Asimismo, con un porcentaje no muy distante se observa un $26.66 \%$ de mujeres entre 30 a 39 años. En menor proporción de participantes se observa el sexo masculino con un rango de edad entre 20 y 29 años $(11.1 \%)$.

\section{Instrumentos}

Escala de Depresión, Ansiedad y Estrés-21(DASS-21; Bados, Solana, y Andrés, 2005): versión abreviada de la escala original de Lovibond y Lovibond 
(Depression, Anxiety, Stress Scales [DASS-21], 1995) para la valoración autoinformada sobre Depresión, Ansiedad y Estrés. Consta de 21 ítems divididos en tres escalas de 7 ítems en cada dimensión, valorando de 0 a 3 la intensidad o frecuencia en que se experimenta cada uno de los síntomas. La subescala que mide la Depresión, considera aspectos relacionados con el bajo afecto positivo como la disforia, desesperanza, tristeza, anhedonia, desvalorización de la vida, auto-desprecio y falta de interés o de implicación. La subescala que evalúa la Ansiedad, comprueba aspectos relacionados con la activación psicofisiológica o la excitación autonómica (sudor de manos, temblor, etc.), y experiencias subjetivas de Ansiedad. Por su parte, la subescala que mide el Estrés, evalúa la dificultad para estar relajado, la excitación nerviosa, la agitación, la irritabilidad y la impaciencia.

Los niveles de fiabilidad de la escala española (Bados et al., 2005) fueron adecuados en todas sus dimensiones ( $\alpha=$ entre .70 y .84).

Maslach Burnout Inventory (MBI; Seisdedos, 1997): versión española adaptada del MBI (Maslach y Jackson, 1986) para evaluar el burnout. Los 22 ítems del cuestionario recogen las tres dimensiones establecidas por los autores como definitorios del síndrome de burnout: Cansancio Emocional, Despersonalización y baja Realización Personal. Se considera que el trabajador presenta mayor burnout cuando informa altas puntuaciones en Cansancio Emocional y Despersonalización y bajas puntuaciones en Realización Personal.

Presenta adecuados niveles de fiabilidad en todas sus dimensiones, tanto para la versión original ( $\alpha=$ entre .74 y .89) como para la adaptada ( $\alpha=$ entre .71 y .90).

\section{Procedimiento}

En primer lugar, se solicitó la autorización a los equipos directivos de los centros, elaborando una carta informativa en la que se explicaba el propósito del estudio y se solicitaba su colaboración a participar en la investigación. Se contó con la ayuda de todos los directores de los respectivos centros participantes. Por lo que, se procedió a la administración del cuestionario vía on-line, de manera voluntaria e individual.

\section{Diseño}

La influencia de la probabilidad de predicción sobre el burnout, concretamente Despersonalización y baja Realización Personal, en presencia de otras variables (Depresión, Ansiedad y Estrés) fue analizada mediante la técnica estadística de regresión logística, siguiendo el procedimiento de pasos hacia delante basado en el estadístico de Wald. El objetivo primordial de esta técnica es cuantificar cómo influye en la probabilidad de aparición de un suceso dicotómico, la presencia o ausencia de diversos factores y el valor de los mismos.

En este caso, el modelo logístico utilizado permite estimar la probabilidad de que se dé un suceso o resultado, por ejemplo, alto o bajo burnout, frente a que no se dé, 
en presencia de uno o más predictores. Esta probabilidad es estimada mediante el estadístico denominado odd ratio (OR), siendo su interpretación de la siguiente manera: si la OR es mayor que uno, por ejemplo 3, por cada vez que se dé el evento en presencia de la variable independiente se dará tres veces si esta variable está presente. Por el contrario, si la OR es menor que uno, por ejemplo 0.5, la probabilidad de que se dé el evento en ausencia de la variable independiente será mayor que en su presencia.

Fueron evaluados la calidad y el ajuste de los modelos propuestos a partir de los siguientes indicadores: la $R^{2}$ de Nagelkerke, que indica el porcentaje de varianza explicada por el modelo (Nagelkerke, 1991) y el porcentaje de casos correctamente clasificados por el modelo o eficiencia pronostica. Puesto que la variable dependiente debe ser dicotómica para su uso en los modelos logísticos esta fue dicotomizada considerando bajas puntuaciones en dicho factor la obtención de un centil inferior a 25 , mientras que altas puntuaciones un centil superior a 75 .

\section{RESULTADOS}

La tabla 2 muestra los resultados de probabilidad de presentar alta Despersonalización en función de la presencia de Depresión, Ansiedad y/o Estrés.

El modelo propuesto permite hacer una estimación correcta para la variable Depresión en el $56.1 \%$ de los casos $\left(\chi^{2}=11.99 ; p<.001\right)$ estos datos permiten afirmar que esta variable puede ser considerada predictora de la alta Despersonalización. Los componentes del modelo expresados por la odd ratio (OR) indican que la probabilidad de presentar alta Despersonalización es 1.07 mayor por cada punto de aumento en la variable Depresión. Es decir, la probabilidad de presentar Despersonalización aumenta un 7\%. En relación con la variable Ansiedad el modelo permite hacer una estimación del $58.8 \%$ de los casos $\left(\chi^{2}=15.70 ; p>.001\right)$. Los componentes del modelo expresados por la odd ratio (OR) indican que la probabilidad de presentar alta Despersonalización es 1.08 mayor por cada punto de aumento en la variable Ansiedad. De este modo, la probabilidad de presentar Despersonalización aumenta un $8 \%$. Por último, en cuanto a la variable Estrés, el modelo permite hacer una estimación del $60.4 \%$ de los casos $\left(\chi^{2}=58.23 ; p<.001\right)$.

Los componentes del modelo expresados por la odd ratio (OR) indican que la probabilidad de presentar alta Despersonalización es 1.15 mayor por cada punto de aumento en la variable Estrés, por lo que la probabilidad de presentar Despersonalización aumenta un $15 \%$.

Es decir, las variables Depresión, Ansiedad y Estrés predicen significativamente altos niveles de Despersonalización en el personal docente nouniversitario. 
GRANADOS et al. Depresión, ansiedad y estrés en despersonalización y baja realización personal

Tabla 2. Regresión logística binaria para la probabilidad de la alta Despersonalización en función de las variables Depresión, Ansiedad y Estrés

\begin{tabular}{|c|c|c|c|c|c|c|c|c|c|c|}
\hline $\mathrm{V}$ & $\mathrm{D}$ & $\%$ & $\chi^{2}$ & $R 2$ & $\mathrm{~B}$ & E.T. & Wald & $p$ & OR & I.C. $95 \%$ \\
\hline \multirow[t]{2}{*}{ D } & $\begin{array}{c}\text { Clasificados } \\
\text { Correctamente }\end{array}$ & 56.1 & 11.99 & .019 & .073 & .022 & 11.40 & .001 & 1.07 & $1.03-1.12$ \\
\hline & Constante & & & & -.101 & .096 & 1.12 & .289 & .90 & \\
\hline A & $\begin{array}{c}\text { Clasificados } \\
\text { Correctamente }\end{array}$ & 58.8 & 15.70 & .025 & .081 & .021 & 14.72 & $<.001$ & 1.08 & $1.04-1.13$ \\
\hline \multirow{3}{*}{$\mathrm{E}$} & Constante & & & & -.111 & .092 & 1.46 & .227 & .89 & \\
\hline & $\begin{array}{c}\text { Clasificados } \\
\text { Correctamente }\end{array}$ & 60.4 & 58.23 & .090 & .139 & .019 & 51.73 & $<.001$ & 1.15 & $1.11-1.19$ \\
\hline & Constante & & & & -.728 & .136 & 28.51 & $<.001$ & .48 & \\
\hline
\end{tabular}

Nota: $\mathrm{V}=$ Variable; $\mathrm{D}=$ Depresión, $\mathrm{A}=\mathrm{Ansiedad} ; \mathrm{E}=$ Estrés; $\chi^{2}=$ Chi cuadrado; $R 2=$ Cuadrado de Nagelkerke; $\mathrm{B}=\mathrm{Coeficiente}$ de regresión; E.T.=Error estándar; Wald=Prueba de Wald; $p=$ probabilidad; OR=Odd ratio; $I$. $C .=$ Intervalo de confianza al $95 \%$

En la tabla 3 se muestra el modelo de regresión logística binaria para la probabilidad de la baja Realización Personal en función de la Ansiedad y el Estrés.

Tabla 3. Regresión logística binaria para la probabilidad de la Realización Personal en función de las variables Ansiedad y Estrés

\begin{tabular}{ccccccccccc}
\hline $\mathrm{V}$ & $\mathrm{D}$ & $\%$ & $\chi^{2}$ & $\mathrm{R} 2$ & $\mathrm{~B}$ & $\mathrm{E} . \mathrm{T}$. & $\mathrm{Wald}$ & $\mathrm{p}$ & $\mathrm{OR}$ & $\mathrm{I} . \mathrm{C} .95 \%$ \\
\hline $\mathrm{A}$ & $\begin{array}{c}\text { Clasificados } \\
\text { Correctamente }\end{array}$ & 60.40 & 62.76 & .09 & -.06 & .03 & 4.53 & .033 & 1.02 & $.88-.99$ \\
\cline { 2 - 10 } & $\begin{array}{c}\text { Clasificados } \\
\text { Correctamente }\end{array}$ & & & .17 & .02 & 43.84 & $<.001$ & 1.19 & $1.13-1.26$ \\
\hline $\mathrm{E}$ & Constante & & -.78 & .13 & 31.70 & $<.001$ & .45 \\
\hline
\end{tabular}

Nota. V=Vaiahles A=Anseddt D=Depesór $\chi^{2}=$ Chi cuadrado; R2=Cuadrado de Nagelkerke; B=Coeficiente de regresión; E.T.=Error estándar; Wald=PruebadeWald;p=probabilidad;OR=Oddratio;I.C.= Intervalodeconfianzaal95\%

Los resultados permitieron hacer una estimación correcta para todas las variables, en un $60.4 \%$ de los casos $\left(\chi^{2}=62.76 ; p<.001\right)$. Los componentes del modelo expresados por la odd ratio (OR) indican que la probabilidad de presentar baja Realización Personal es mayor para ambas variables por cada punto de aumento, concretamente, 1.02 en Ansiedad y 1.19 en Estrés respectivamente (ver Tabla 3).

\section{DISCUSIÓN Y CONCLUSIONES}

El presente estudio tuvo como objetivo analizar, mediante análisis de regresión logística, si la Depresión, la Ansiedad y el Estrés son predictores de altos niveles de Despersonalización y baja Realización Personal en docentes de EI, EP y ESO.

Los resultados del presente estudio revelan que un mayor nivel de Depresión, Ansiedad y/o Estrés predicen mayores niveles de Despersonalización. Concretamente, la probabilidad de presentar Despersonalización aumenta un 7\% por cada punto de aumento de la Depresión, en una estimación del $56.1 \%$ de los casos; $8 \%$ por cada punto de aumento de la Ansiedad en una estimación del $58.8 \%$ de los casos; y un $15 \%$ por cada punto que se incrementa el Estrés, en una estimación del $60.4 \%$ de los casos. La capacidad explicativa 
del modelo, observada en nuestro estudio en esta dimensión, fue bastante superior a la capacidad explicativa de los modelos de Correia, Gomes, y Moreira (2010), con una capacidad predictiva de $45 \%$, así como la observada por Arias-Galicia y GonzálezZermeño (2009), Moreno-Jiménez, González, y Garrosa (2001), y Pinto, De Almeida, y Miyazaki (2010) con similar valor predictivo (42\%).

Asimismo, los resultados obtenidos están en consonancia con investigaciones previas. Profesores susceptibles al Estrés evidencian índices altos de Despersonalización (Correia et al., 2010; Howlett et al., 2015; Maslach y Schaufeli, 1993; Moreno-Jiménez et al., 2001; Pinto et al., 2010; Rui-Gomes, Montenegro, Da Costa-Peixoto, y Da CostaPeixoto, 2010), y maestros con mayores niveles de Ansiedad, también, sufren mayores niveles de Despersonalización (Yu, Wang, Zhai, Dai, y Yang, 2015).

Por otra parte, la probabilidad de presentar baja Realización Personal, según los hallazgos del presente estudio, aumenta un $2 \%$ por cada punto que se eleva la Ansiedad, en una estimación del $60.40 \%$ de los casos; y un $19 \%$ por cada punto que se incrementa el Estrés. Investigaciones previas afirman nuestros resultados. Moreno-Jiménez et al. (2001) observaron que la baja Realización Personal es predictora del Estrés de rol. Rui-Gomes et al. (2010) afirmaron que la baja Realización Personal predice una combinación de factores estresantes. Por su parte, Moriana-Elvira y Herruzco (2005) afirmaron que las diferentes variables del burnout predicen diversas variables psicológicas entre las que se encuentran la Depresión y la Ansiedad.

Desde otra perspectiva, los hallazgos encontrados muestran cierta controversia con investigaciones previas evaluadas con participantes de otros países, observando correlaciones negativas de Despersonalización con Ansiedad y Depresión en docentes colombianos (N=76 maestros) (Fernández, 2017). A este respecto, cabe destacar diversas limitaciones en el estudio. En primer lugar, estos resultados no pueden generalizarse a otros colectivos culturales. Asimismo, debería evaluarse el estudio con muestra participante de diversa profesión. En este sentido, Howlett et al. (2015), con profesionales de la salud ( $\mathrm{N}=616$ médicos canadienses), expresaron que respuestas del Estrés emocional era lo que predecía mayores niveles de Despersonalización y baja Realización Personal. Sin embargo, futuras líneas de investigación podrían replicar el estudio en profesionales de otros ámbitos para observar la diversidad de resultados.

Asimismo, es importante evaluar la relación entre las variables estudiadas teniendo en cuenta la diversidad sexual y la experiencia laboral. Existen estudios que demuestran que el estrés laboral, con mayor proporción en el sexo masculino, son predictores de la Despersonalización en docentes canadienses (Burke, Greenglass, y Schwarzer, 1996), venezolanos (Oramas-Viera et al., 2007), noruegos (Skaalvik y Skaalvik, 2017), así como israelíes (Naz, Atta, y Iqbal-Malik, 2017). Del mismo modo, el sexo masculino, en muestra canadiense obtiene mayor presencia de baja Realización Personal que el sexo femenino (Howlett et al., 2015). Por otra parte, docentes de Colombia 
( $\mathrm{N}=82$ docentes) presentaron normales niveles de Despersonalización, en su gran mayoría, obteniendo una relación no significativa entre variables de sexo, edad y experiencia laboral, entre otras (Díaz-Bambula et al., 2012).

Del mismo modo, estos resultados no deben ser generalizados en docentes que ejerzan su profesión en distinta etapa a la investigada en el presente estudio. Una posible línea de investigación futura podría observar las diferencias que presentan docentes universitarios.

En conclusión, las variables Depresión, Ansiedad y Estrés predicen significativamente altos niveles de Despersonalización y baja Realización Personal en docentes de EI, EP y ESO. Este hallazgo favorece una visión específica sobre dimensiones de burnout y el elevado grado de comorbilidad con trastornos emocionales que pueden llegar a presentar los profesionales de la Educación. A este respecto, es destacable observar como diversos estudios muestran una tasa considerable de Despersonalización. Un estudio con docentes colombianos ( $\mathrm{N}=239 ; M$ edad=42 años) demostraron en el $34 \%$ de los participantes altos (12\%) y medios niveles (21\%) de Despersonalización (RestrepoAyala, Colorado-Vargas y Cabrera-Arana, 2006). Niveles similares (38.2\%) observó Fernández (2017) con profesorado del mismo país.

Este estudio permite la evaluación predictiva de las variables mencionadas, así como información para diseñar programas de intervención con el fin de disminuir conductas desadaptativas, que de no acotarse pueden originar problemáticas reflejadas en la calidad del aprendizaje e interacción con el alumnado. Pues, como dejan entrever Moriana-Elvira y Herruzco (2005), cabría preguntarse si la aparición del burnout, y en este caso concreto de la Despersonalización y la baja Realización Personal es un precedente de las variables psicológicas estudiadas o son estas últimas quien determinan ciertas o todas las dimensiones del burnout. De lo que se tiene certeza es que sea cual sea la causa, las consecuencias son dañinas tanto para el bienestar emocional del profesorado como la calidad de la enseñanza del alumnado.

\section{REFERENCIAS}

Arias-Galicia, F., y González-Zermeño, M.E. (2009). Estrés, agotamiento profesional (burnout) y salud en profesores de acuerdo a su tipo de contrato [Stress, burnout and health in teachers according to their type of contract]. Ciencias y Trabajo, 11(31), 172-176.

Bados, A., Solanas, A., y Andrés, R. (2005). Psychometric properties of the Spanish version of depression, anxiety and stress scales (DAS). Psicothema, 17(4), 679-683.

Burke, R.J., Greenglass, E.R., y Schwarzer, R. (1996). Predicting teacher burnout over time: effects of work stress, social support, and self-doubts on burnout and its consequences. Anxiety, Stress y Coping: An International Journal, 3, 261-275.

Cladellas, R., y Castelló, A. (2011). Percepción del estado de salud y estrés de profesorado universitario en relación con la franja horaria de docencia. Electronic Journal of Research in Educational Psychology, 9(1), 217-240. 
Cockerham, W.C. (2001). Handbook of medical sociology. Nueva York: Prentice-Hall.

Cornejo-Chávez, R., y Quiñónez, M. (2007). Factores asociados al malestar/bienestar docente. Una investigación actual. REICE: Revista Electrónica Iberoamericana sobre Calidad, Eficacia y Cambio en Educación, 5(5), 75-80.

Correia, T., Gomes, A.R., y Moreira, S. (2010). Stresse ocupacional em profesores do ensino básico: um estudo sobre as diferenças pessoais e profissionais. In C. Nogueira, I. Silva, L. Lima, A.T. Almeida, R. Cabecintas, R. Gomes, C. Machado, A. Maia, A. Sampaio, y, M.C. Taveira (Eds.), Actas VII Simpósio Nacional de Investigação em Psicologia (pp.14771493). Lisboa: Associação Portuguesa de Psicologia.

Díaz-Bambula, F., López-Sánchez, A.M., y Varela-Arévalo, M.T. (2012). Factores asociados al síndrome de burnout en docentes de colegios de la ciudad de Cali, Colombia [Factors associated to burnout syndrome in high school teachers in Cali, Colombia]. Universitas Psychologica, 11(1), 217-227.

Fernández, M.P. (2017). Relación entre desgaste ocupacional y manifestaciones psicosomáticas en una muestra de docentes colombianos de la ciudad de Santa Marta. Archivos de Medicina, 17(1), 78-90.

Figueiredo-Ferraz, H., Gil-Monte, P.R., Grau-Alberola, E., Llorca-Pellicer, M., y García-Juesas, J.A. (2012). Influence of some psychosocial factors on mobbing and its consequences among employees working with people intellectuall disabilities. Journal of Applied Research in Intellectual Disabilities, 25(5), 455-463.

Frenzel, A.C., Pekrun, R., Goetz, T., Daniels, L.M., Durksen, T.L., Becker-Kurz, B., y Klassen, R.M. (2016). Measuring teacher's enjoyment, anger, and anxiety: the teacher emotions scales (TES). Contemporany Educational Psychology, 46, 148-163.

Grau, A., Suñer, R., y García, M.M. (2005). Desgaste profesional en el personal sanitario y su relación con los factores personales y ambientales [Burnout syndrome in health workers and relationship with personal and environmental factors]. Gaceta Sanitaria, 19(6), 463470.

Howlett, M., Doody, K., Murray, J., Le-Blanc-Duchin, D., Fraser, J., y Atkinson, P.R. (2015). Burnout in emergency department healthcare professionals is associated with coping style: a cross-sectional survey. Emergency Medicine Journal, 32(9), 722-727.

Husain, W., Gulzar, A., Aqueel, M., y Rana, A. (2016). The mediating role of depression, anxiety and stress between job strain and turnover intentions among male and female teachers. Journal of Social Sciences, 10(1), 48-57.

Llorca-Rubio, J.L., y Gil-Monte, P.R. (2014). Prevención de riesgos laborales y su relación con el género de los trabajadores [Occupational risks prevention and their relationships to workers' gender]. Saúde e Sociedade, 22(3), 727-735.

Lovibond, P.F., y Lovibond, S.H. (1995). The structure of negative emotional states. Comparison of the Depression Anxiety Stress Scales (DAS) with the Beck Depression and Anxiety Inventories. Behaviour Research and Therapy, 33(3), 335-343.

Maslach, C., y Jackson, S.E. (1986). Maslach Burnout Inventory ( $2^{\mathrm{a}} \mathrm{ed}, 19811^{\mathrm{a}}$ ed.). Palo Alto, California: Consulting Psychologists Press

Maslach, C., y Schaufeli, W.B. (1993). Historical and conceptual development of burnout. En W. Schaufeli, C. Maslach, y T. Marek (Eds.), Professional burnout:recent developments in theory and research (pp.1-16). Washington: DC. Taylor y Francis.

Mechanic, D. (1976). Stress illness and illness behavior. Journal of Human Stress, 2(2), 2-6.

Moreno-Jiménez, B., González, J.L., y Garrosa, E. (2001). Desgaste profesional (burnout), personalidad y salud percibida. En J. Buendía y F. Ramos (Eds.), Empleo, estrés y salud (pp.59-83). Madrid: Pirámide. 
Moriana-Elvira, J.A., y Herruzco, J. (2005). El síndrome del burnout como predictor de bajas laborales de tipo psiquiátrico. Clínica y Salud, 16(2), 161-172.

Nagelkerke, N.J.D. (1991). A note on a general definition of the coefficient of determination. Biometrika, 78(3), 691-692.

Naz, F., Atta, M., y Iqbal-Malik, N. (2017). Teacher self-efficacy as determining factors of burnout among college teachers. Journal of Research in Social Sciences 5(2), 105-120.

Oramas-Viera, A., Almirall-Hernández, P., y Fernández, I. (2007). Estrés Laboral y el Síndrome de Burnout en Docentes Venezolanos. Salud de los Trabajadores, 15(2), 71-87.

Pinto, D.R., de Almeida, T.E.P., y Miyazaki, M.C.O.S. (2010). A saúde e estresse ocupacional em médicos. Arquivos de Ciências da Saúde, 17(4), 201-205.

Restrepo-Ayala, N., Colorado-Vargas, G.O., y Cabrera-Arana, G.A. (2006). Desgaste emocional en docentes oficiales de Medellín, Colombia, 2005. Revista de Salud Pública, 8(1), 63-73.

Rui-Gomes, A.R., Montenegro, N., Da Costa-Peixoto, A.M.B.C., y Da Costa-Peixoto, A.R.B.C. (2010). Stress ocupacional no ensino: um estudo com professores dos $3^{\circ}$ ciclo e ensino secundário [Occupational stress in teaching: a study with high school teachers]. Psicologia y Sociedade, 22(3), 587-597.

Salanova, M., Llorens, S., y García-Renedo, M. (2003). ¿Por qué se están “quemando" los profesores? Prevención, trabajo y salud, 28, 16-20.

Seisdedos, N. (1997). MBI Inventario Burnout de Maslach. Madrid: Tea Ediciones.

Siegrist, J., Siegrist, K., y Weber, I. (1986). Sociological concepts in the etiology of chronic disease:the case of ischemic herat disease. Social Science and Medicine, 22(2), 247- 253.

Skaalvik, E.M., y Skaalvik, S. (2011). Teacher job satisfaction and motivation to leave the teaching profession: relations with school context, feeling of belonging, and emotional exhaustion. Teaching and Teacher Education, 27(6), 1029-1038.

Skaalvik, E.M., y Skaalvik, S. (2016). Teacher stress and teacher self-efficacy as predictors of engagement, emotional exhaustion, and motivation to leave the teaching profession. Creative Education, 7(13), 1785-1799.

Skaalvik, E.M., y Skaalvik, S. (2017). Dimensions of teacher burnout: relations with potential stressors at school. Social Psychology of Education, 20(4), 775-790. Turner, R. J., Wheaton, B., y Lloyd, D.A. (1995). The epidemiology of social stress. American Sociological Review, 60(1), 104-125.

Vera, M., Salanova, M., y Martín, B. (2010). Profesorado universitario y su bienestar laboral. La importancia del triple perfil laboral. Electronic Journal of Research in Educational Psychology, 8(2), 581-602.

Yu, X., Wang, P., Zhai, X., Dai, H., y Yang, Q. (2015). The effect of work stress on job burnout among teachers: the mediating role of self-efficacy. Social Indicators Research, 122(3), 701-708.

Recibido: 23 de abril de 2019 Recepción Modificaciones: 30 de abril de 2019

Aceptado: 5 de mayo de 2019 